\title{
Towards virtual machine introspection based security framework for cloud
}

\author{
BHAVESH BORISANIYA* and DHIREN PATEL \\ Department of Computer Engineering, Sardar Vallabhbhai National Institute of Technology Surat, \\ Surat 395007, India \\ e-mail: borisaniyabhavesh@gmail.com
}

MS received 19 August 2017; revised 14 June 2018; accepted 27 August 2018; published online 25 January 2019

\begin{abstract}
Virtualization enables provision of resources to users according to their requirement through Infrastructure as a Service (IaaS) delivery model in cloud computing environment. Malicious users could lease cloud resources and use them as platforms to launch attacks. In this paper, we propose a Virtual Machine Introspection (VMI)-based security framework to monitor cloud users' in-VM activities and detect malicious one if any. We justify our selection of VMI method based on hardware knowledge for proposed framework by discussing its key advantages over other VMI methods. We propose design of multi-threaded analysis component that can introspect number of virtual machines hosted on cloud servers in real time. Experimental results demonstrate that our framework performs well with a set of metrics appropriate for cloud IaaS environment.
\end{abstract}

Keywords. Virtual Machine Introspection; cloud computing; vector space model; system call trace; malware.

\section{Introduction}

Cloud computing refers to amalgamation of known technologies (virtualization, internetworking, utility computing, etc.), utilized to manage and deliver services over the Internet. Infrastructure as a Service (IaaS) is a key service among various services delivered by cloud. IaaS is driven by virtualization, which allows sharing pool of cloud resources among tenants. Multi-tenancy enables cloud providers to deliver services at lower cost and with less managerial efforts.

Low rental and cheaper services attract attackers to host their malicious code over the cloud in order to disrupt the cloud services or to target other cloud users [1, 2]. For example, attackers can host botnet over the cloud virtual machines (VMs) and use them to target victim(s) through command and control (C\&C) [1]. Disruption of service (generated from same cloud resources) affects credibility of cloud service (and of providers). Cloud providers need a strong in-VM activity detection system to monitor the VM (from outside) at hypervisor layer. Virtual Machine Introspection (VMI) is a promising technique to achieve the task at hand [3].

VMI gives inside view of VM by analysing its system states, which comprise software state and hardware state, from outside VM [4]. The key challenge with VMI is to interpret high-level information (e.g., processes and files) from low-level information (e.g., register values, system

*For correspondence calls, I/O requests, etc.) extracted from outside. This gap between actual states inside the VM and relevant information interpreted from outside the VM is known as semantic gap.

In order to bridge semantic gap, Pfoh et al [4] have defined three view generation delivery patterns for VMI, namely, in-band, out-of-band and derivation.

In-band delivery pattern requires an agent to be placed (or hooked) inside the guest operating system (OS). It extracts the required details and sends them to its counterpart running in host OS. It provides a comprehensive inner view; however, it is still liable to be easily bypassed by terminating (or killing) agent service by the user (who owns that VM).

Out-of-band delivery pattern relies upon the external semantic knowledge of OS. It uses the kernel symbol table to find the location of key data structures of kernel in order to find in-VM information from VM memory dump (or hard disk image). As it is highly dependent on kernel data structure, VMI systems based on out-of-band delivery pattern are vulnerable to Dynamic Kernel Structure Manipulation (DKSM) attack [5], in which the attacker can dynamically modify fields of kernel data structures to subvert VMI component.

Compared with previous two delivery patterns, derivation-based delivery pattern has narrow inner view of VM. It counts on the hardware knowledge of the VM and monitors the registers, interrupts, etc. for VM introspection. An attacker cannot hide its presence from underlying hardware and hence, derivation is the most reliable technique for 
VMI. It is guest OS portable, and does not rely on software architecture. Also, as it utilizes only hardware state information that cannot be evaded by attackers, it remains evasion resistant [4].

We propose a VMI-based security framework that leverages the derivation-based approach to monitor activities running inside the VM from outside by extracting the system call traces of each process and detect malicious activities if any. We utilize the hardware-based system call tracing tool Nitro [6] to extract system call traces of in-VM processes.

Design of our framework was proposed earlier in [7]. Later we also proposed a modified vector space representation model in [8] to build feature vector for classification of processes into benign and malicious. The modified vector space model is capable of handling any unforeseen system calls that were not considered during the training phase. In addition to this, our detection framework can cope up with the scalability of cloud environment.

In this paper, we introduce the design of Analyzer - a core component of VMI-based security framework architecture. Analyzer utilizes the modified vector space representation [8] to model behaviour of processes. It uses the multi-threading capability to handle events from multiple cloud nodes hosting multiple VMs. We also provide the evaluation of Analyzer on the basis of a set of real-time metrics.

Major contribution of this work is listed as follows:

- proposed the design of a multi-threaded Analyzer component (suitable for cloud environment) to analyse VMs' activities;

- derived metrics to evaluate performance of proposed multi-threaded Analyzer;

- developed a prototype and experimented with known system call trace datasets.

Rest of the paper is organized as follows. Section 2 describes related work found in the literature. Architecture design, system call trace representation and working of proposed framework are discussed in section 3. Section 4 converses experiments and analysis of results with conclusion and references at the end.

\section{Background and related work}

\subsection{Virtual machine introspection}

Virtual machine introspection (VMI) is mainly used in forensic analysis and in security applications like an intrusion detection system (IDS). There are numerous approaches proposed to bridge the semantic gap, the biggest hurdle in applicability of VMI.

Virtuoso [9], XenAccess [10], LibVMI [11] and InSight [12] are approaches that can be considered as VMI libraries, used to interpret the high-level in-VM information from raw level data. These libraries are used as the core VMI component in applications for extracting VM state information, whereas other functionalities are built on top of them.

XenAccess, LibVMI and Insight are based on out-ofband delivery pattern, which utilizes a kernel symbol table to map key data structures to extract particular information.

Virtuoso on the other hand initially uses an agent program running inside the guest OS (in-band delivery pattern) to generate an external introspection program that can run and extract in-VM information from outside the guest OS. Virtuoso can generate introspection program only outside VM for application that has been executed and trained within VM. To overcome this limitation, Fu and Lin [13] proposed Virtual Machine Space Traveller (VMST). VMST redirects kernel data access under the execution context of system calls of interest without any training and uses dynamic taint analysis approach [14] to locate the kernel data to be redirected for introspection. Dynamic taint analysis used in VMST hampers the performance of the system [15]. HYBRID-BRIDGE [15] proposed by Saberi et al is built on top of VMST. It decouples the online taint analysis used by VMST, with online memoization [16] of trained meta-data. HYrBRID-BRIDGE introspects the VM memory and manages VM introspection through two trusted VMs: FASt-BRidge and Slow-BRidge. Slow-Bridge extracts the meta-data using kernel data redirection and passes trained meta-data using memoization for fast memory introspection to FAST-BRIDGe. Hybrid-BRIDGe requires the same OS version to be installed in trusted VMs as that of user VM under introspection. All these approaches are inherently dependent upon the kernel data structure to acquire relevant information and susceptible to DKSM attack.

There are other approaches like Ether [17] and Nitro [6] that use derivation-based view generation pattern for information extraction. Ether is a malware analysis approach, and works with Xen hypervisor. It supports instructions and system calls tracing of guest OS. The creator of Ether has stopped updating it [18]. Nitro on the other hand is a hardware-based system call tracing tool that extends the Kernel Virtual Machine (KVM) to support trapping of system interrupts and user interrupts into hypervisor.

Related approaches reported in literature are briefly discussed as follows. CloudSec [19], proposed by Amani S Ibrahim et al, is a virtualization-aware monitoring appliance, which provides real-time introspection for hosted VMs in the Cloud IaaS model. It provides protection against kernel data rootkits. CloudSec is built on top of VMware VMsafe [20] API framework. It is used for VM introspection and works with VMware-based hypervisor only. Baek et al [21] have made it possible to provide VMI as a service through their approach named as CloudVMI. CloudVMI uses LibVMI on Xen hypervisor to serve VMI 


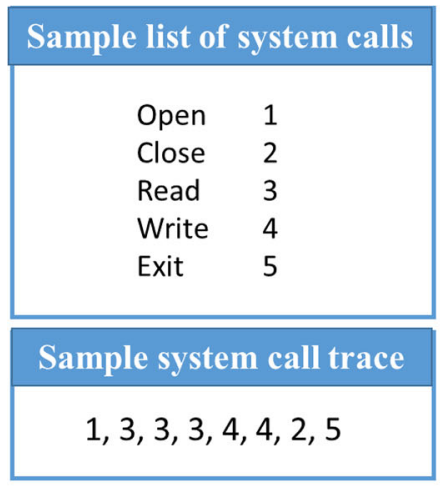

(a)

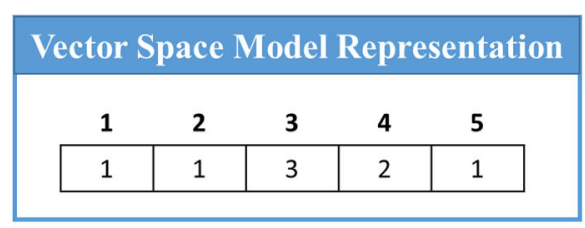

(b)

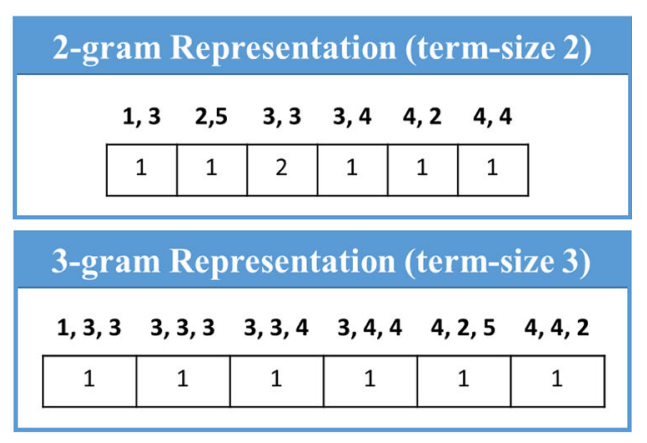

(c)

\begin{tabular}{|c|c|c|c|c|c|c|c|c|c|c|c|c|c|c|c|c|c|c|c|c|c|c|c|c|}
\hline \multicolumn{25}{|c|}{ 2-gram Representation (term-size 2) <Total 25 terms > } \\
\hline 1,1 & 1,2 & 1,3 & 1,4 & 1,5 & 2,1 & 2,2 & 2,3 & 2,4 & 2,5 & 3,1 & 3,2 & 3,3 & 3,4 & 3,5 & 4,1 & 4,2 & 4,3 & 4,4 & 4,5 & 5,1 & 5,2 & 5,3 & 5,4 & 5,5 \\
\hline 0 & 0 & 1 & 0 & 0 & 0 & 0 & 0 & 0 & 1 & 0 & 0 & 2 & 1 & 0 & 0 & 1 & 0 & 1 & 0 & 0 & 0 & 0 & 0 & 0 \\
\hline \multicolumn{25}{|c|}{ 3-gram Representation (term-size 3) < Total 125 terms $>$} \\
\hline 1,1 & & 1,2 & $1,1,3$ & 1,1 , & & $1,1,5$ & 1,2 & & & $3,3,3$ & 3,3 , & & $, 3,5$ & & 4,4 & 4,1 & $4,4,2$ & $4,4,3$ & & & $5,5,3$ & 5,5 & 4 & $5,5,5$ \\
\hline 0 & & 0 & 1 & 0 & & 0 & 0 & & $\ldots .$. & 1 & 1 & & 1 & ........ & 0 & & 0 & 0 & & ......... & 0 & 0 & & 0 \\
\hline
\end{tabular}

(d)

Figure 1. (a) Sample list of system calls and sample system call trace, (b) vector space model representation of sample system call trace, (c) unique terms of term-size 2 and term-size 3 from sample system call trace and (d) all possible terms of term-size 2 and termsize 3 from sample system call trace.

as a service to cloud users. Cloud users can monitor their respective user VMs on cloud through monitoring VM, which contains the virtualized interface of LibVMI (vLibVMI). vLibVMI works as a client library in monitoring VM, connected with server module running in host machine. With the motivation of securing users' privacy, while applying VMI in cloud, Yao et al [22] proposed CryptVMI - an encrypted VMI system for cloud. CryptVMI utilizes LibVMI for VM monitoring. Suneja et al [23] proposed Near Field Monitoring (NFM), a nonintrusive, out-of-band and out-of-the-box system monitoring approach for cloud. NFM provides subscription of outof-the-box monitoring and analytics of users' VMs by delivering Analytics as a Service (AaaS). For extracting VM state information, NFM depends on kernel data structures that may vary across OS versions; however, it is undocumented for certain OS.

\subsection{System call trace representation}

In our proposal, we use Nitro [6] at host machines to extract system call traces of processes running inside the VMs. To classify the process behaviour, we need to derive features from process system call traces, which are received from each cloud node.
The simplest form of system call trace representation is viewing it as a string and matching the subsequence of system call trace in question for its classification. Forrest et al [24] used fixed length system call sequences for anomaly detection. Pfoh et al [25] used such a representation along with String Subsequence Kernel (SSK) [26] and Support Vector Machine (SVM) for malware classification. However, for a live detection system, its overall subsequence matching time complexity turns out to be $\mathrm{O}(n|S W| S V|| s|| t \mid)$, where $s$ and $t$ are two strings to be compared, $|S V|$ is number of support vectors and $|S W|$ is number of system calls considered at a time in a window. As number of processes increases, this becomes ill-suited for an online detection system in cloud framework.

Researchers have also used information-retrieval-based models like Boolean model and vector space model to represent system call information. In terms of information retrieval, the document is relevant if it contains the index terms. A Boolean model is an exact match model, which matches the query with document and results into either 1 (match) or 0 (not match). Wang et al [27] uses $n$-grams, considered as features of Boolean model with SVM and Gaussian Radial Basis Function (GRBF) for classification of worms. The $n$-gram helps in preserving sequence information to some extent. For experimentation, Wang et al have considered 722 benign executables and 1589 


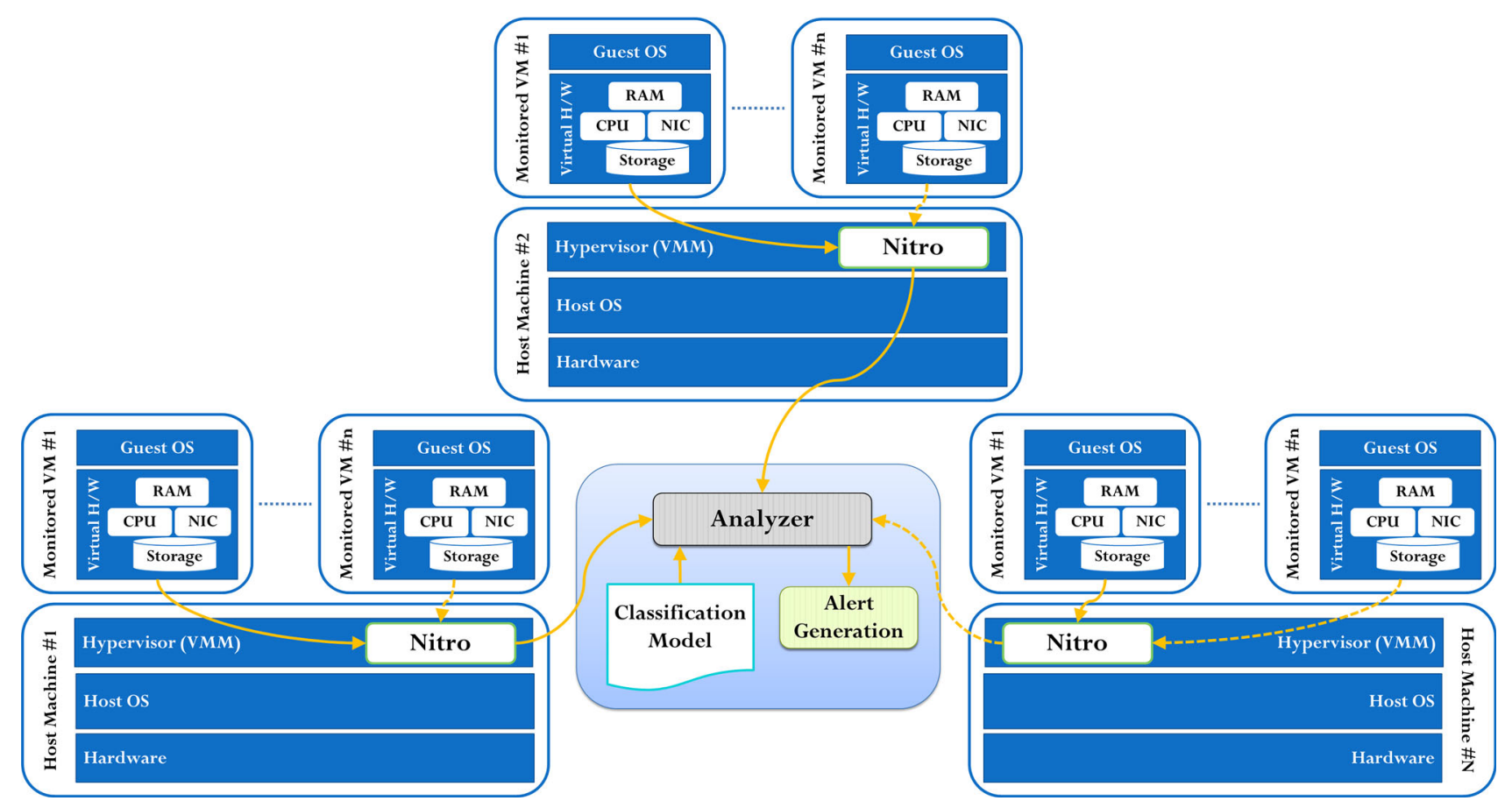

Figure 2. System architecture.

worms. However, their classifier appears to be helpless in correctly classifying other types of malware families.

A Boolean model considers all features as equally important. A vector space model, on the other hand, gives weightage to individual features (index terms) by keeping record of their occurrence in a given document. It represents each document and query as a vector. Each dimension of the vector corresponds to a feature (or term) in vocabulary. If the term appears in a given document, a weight will be assigned to its corresponding dimension in vector. Kang et al [28] firstly proposed 'bag of system calls' and applied vector space model on system call traces. Rieck et al [29] have considered the frequency of system call (occurrence of system call in the document) in a trace as a weight of particular system call and utilized such feature vector to classify malware with Polynomial kernel function. Liao and Vermuri [30] also leveraged the vector space model with $k$-nearest neighbour $(k \mathrm{NN})$ classifier for malware classification, where nearness was calculated with cosine similarity measure. However, vector space model does not preserve sequence information. For example, feature vectors using vector space model for sample system call traces $S_{1}$ : open, read, close, exit and $S_{2}$ : open, exit, close, read are similar, although they have different system call orderings. Losing system call ordering information leaves a system vulnerable to Mimicry Attacks [31, 32], where a malware writer interleaves malware system call trace patterns with benign system call traces.

In our proposal, we define a set of system calls as a term and number of system calls in a term as its term-size. The term is made up of consecutive system calls for the given trace, whose length is equal to the term-size. Such a term defines a feature for process classification. The term-size plays a crucial role in describing the process behaviour. By increasing term-size, one can preserve system call sequence information, which helps in describing the behaviour more accurately. However, increasing term-size suffers with curse-of-dimensionality issue, because, as term-size increases, number of feature dimensions also increases. High-dimension feature space is difficult to handle, because it requires high amount of storage and processing time.

Figure 1 shows the different possible representations for sample system call trace based on vector space model. Figure 1a shows an example sample trace. Figure $1 \mathrm{~b}$ shows the representation using a vector space model, where each index of a vector represents the frequency of each system call (term-size 1) in a given trace. However, the vector space model loses the sequence information of system calls. To preserve the sequence information, we can utilize $n$ gram approach and consider $n$ number of system calls in a term rather than a single system call. Figure 1c shows the vector space model with possible $n$-gram terms of term-size 2 and term-size 3 built from a given system call trace. However, such a representation cannot cover all possible sequences, as it is quite possible that certain system calls are not present in a given system call trace during training, but can be found later in the live system. The solution for this could be consideration of all possible sequences of a given term-size, which can be built using a combination of all system calls given in a list. Figure 1d shows such a 

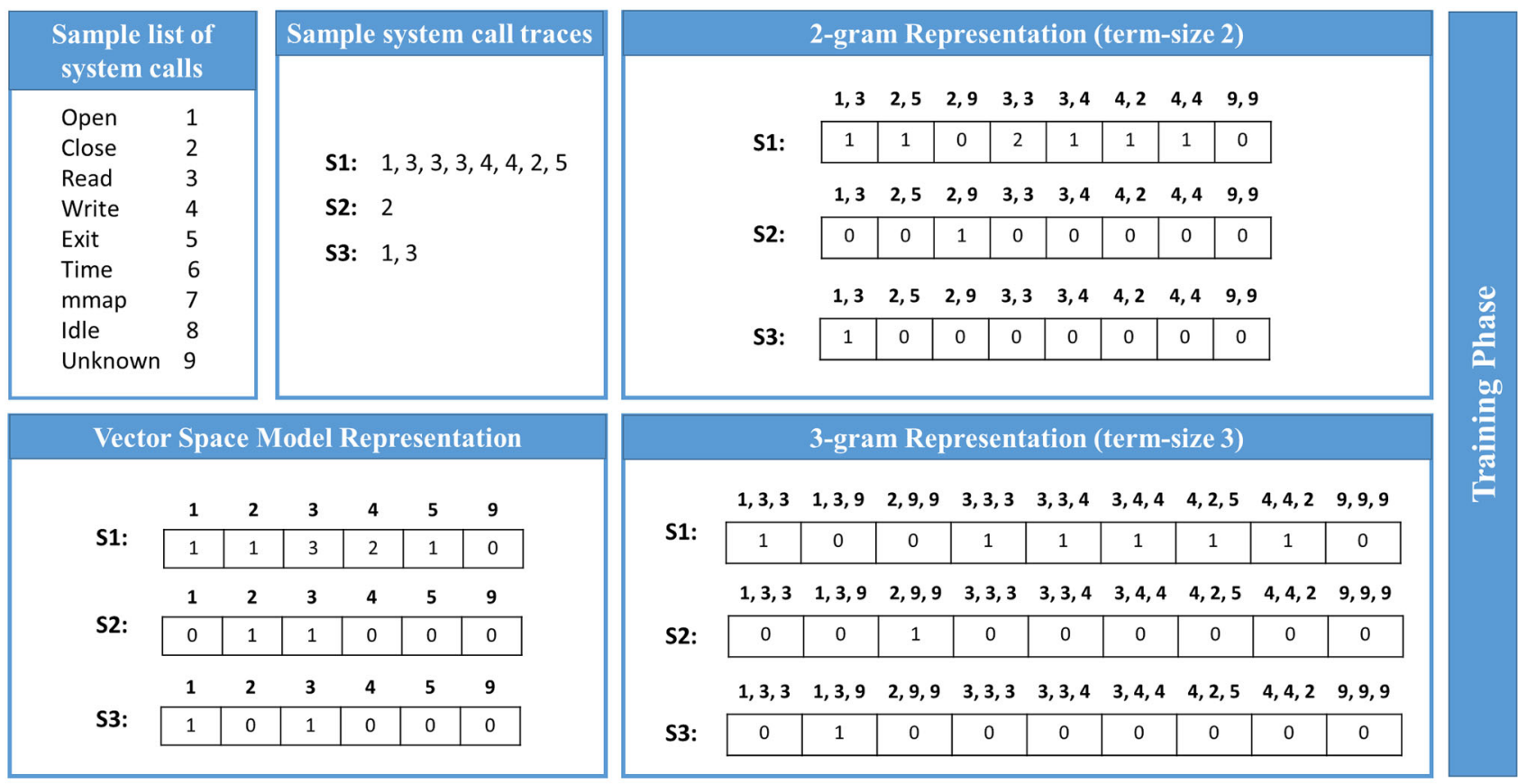

(a)

\begin{tabular}{|lc|}
\hline \multicolumn{2}{|c|}{$\begin{array}{c}\text { Sample list of } \\
\text { system calls }\end{array}$} \\
\hline Open & 1 \\
Close & 2 \\
Read & 3 \\
Write & 4 \\
Exit & 5 \\
Time & 6 \\
mmap & 7 \\
Idle & 8 \\
Unknown & 9 \\
\hline
\end{tabular}
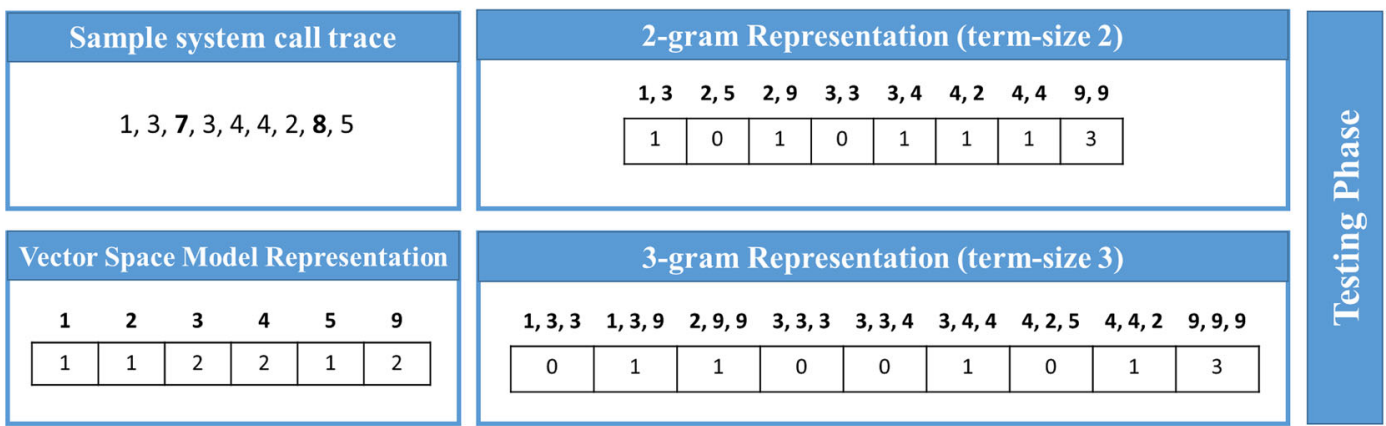

(b)

Figure 3. Feature terms in vector space model, modified vector space representation (term-size 2 and term-size 3) for sample system call trace with inclusion of unknown terms during (a) training phase and during (b) testing phase.

$\begin{array}{lll}\text { 172.16.10.100 } & \mathbf{1 1 : 0 x 3 4 8 5 B 0 0 0 : 0 : 0 x 3 4 F B 2 0 6 7} & \mathbf{7 8} \\ \text { IP Address } & 172.16 .10 .100 \\ \text { VM ID } & 11 \\ \text { Process ID } & 0 \times 3485 \mathrm{~B} 000: 0: 0 \times 34 \mathrm{FB} 2067 \\ \text { System Call } & 78\end{array}$

Figure 4. Message format received by Analyzer.

representation. For a given sample list of 5 system calls, this represents vector of $5^{2}=25$ terms for term-size 2 and $5^{3}=125$ for term-size 3 . If we consider 250 different system calls in any OS, then it requires $6.25 \times 10^{4}$ terms for term-size 2 and $15.625 \times 10^{6}$ terms for term-size 3 to be stored in a vector. Storing and processing such a huge vector is a difficult task. Hence, we use a modified vector space representation as described in [8]. The modified vector space representation includes $n$-gram sequences and inserts terms based on a deliberately included special system call to handle any unforeseen terms. We will discuss this modified vector space representation in section 3.2.

\section{VMI based security framework for cloud}

\subsection{Architecture}

As shown in figure 2, architecture of our framework contains a hardware-based system call tracing tool (Nitro), placed at hypervisor layer. Nitro extracts the system calls of all the processes running inside all VMs, 


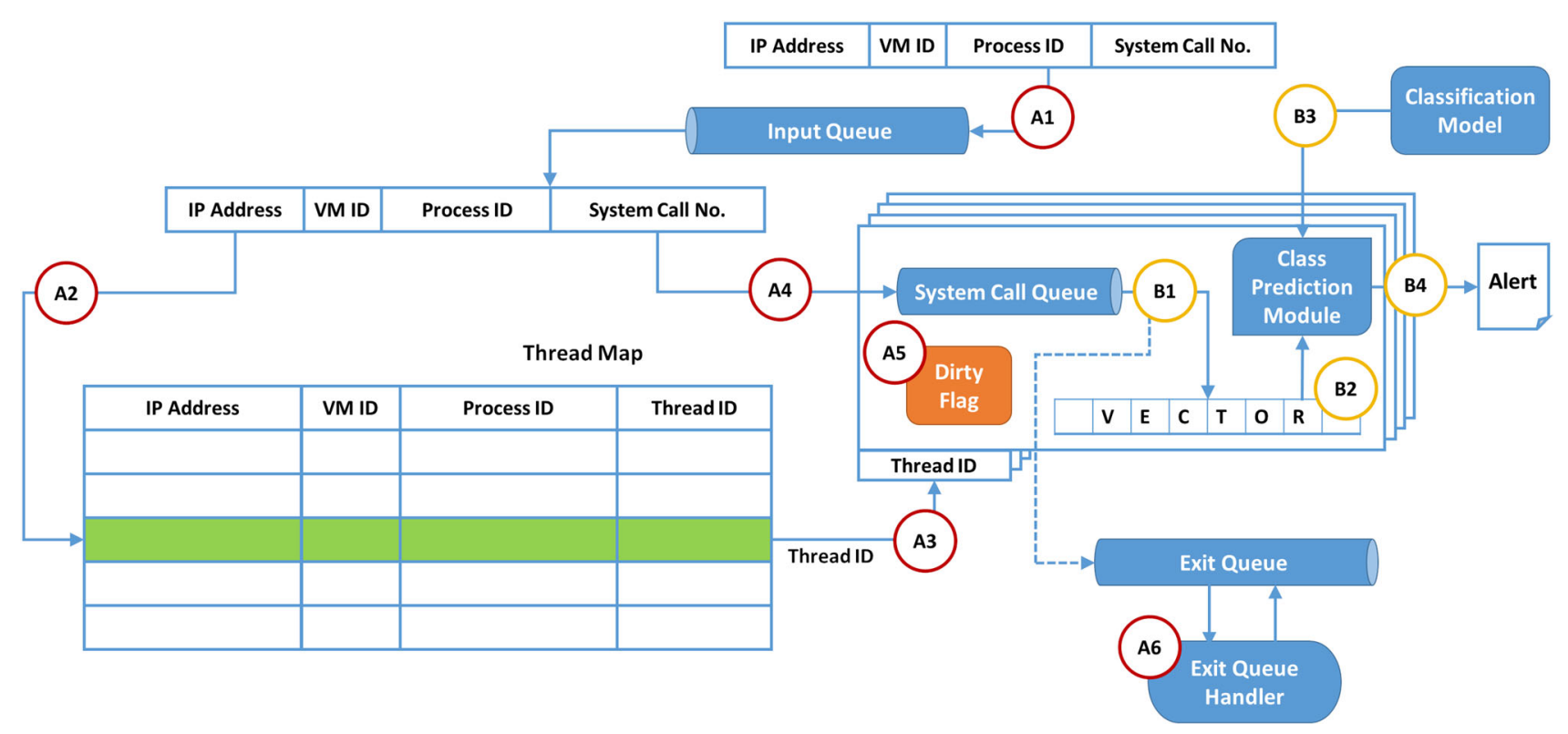

Figure 5. Analyzer - internal working of IP-thread and process-thread.

which are hosted on top of hypervisor. Nitro leverages derivation-based VMI technique to extract the system call information. Extracted system call information is forwarded to the central Analyzer. The Analyzer parses the system call information received from various hosts. It then segregates and maintains the information in terms of system call trace for a particular process, running in a particular VM hosted on a particular host. Such a system call trace is then converted into feature vector using modified vector space representation. After this, with the help of a trained classification model, the Analyzer classifies the process as benign or malicious. If the process is found to be malicious, it will generate an alert.

\subsection{Modified vector space representation}

As discussed earlier, the system call representation as shown in figure 1c is compact, but it cannot map any unforeseen terms. On the contrary, the representation in figure $1 \mathrm{~d}$ is complete, but it grows exponentially with the term-size and hence requires huge storage space and processing time. An intermediate way to handle the situation is to use the modified vector space representation as shown in figure 3. It includes a special system call - unknown (or unk) - deliberately added to the OS system call list. The unknown system call has a number that is higher than other system call numbers. The representation uses terms created with the unknown system call to map any unforeseen terms found during testing phase.

As shown in figure 3a, during training phase, we find all possible unique terms of term-size length from system call traces. If any system call trace contains insufficient number of system calls to form the term of length term-size, the unknown system call number is inserted to form a complete term. Also, one term having all unknown system call numbers of length term-size is included in feature list to map any unforeseen terms found during testing phase. As shown in figure $3 \mathrm{a}$, we include system call number 9 as unknown system call and create certain terms to complete the term-size requirement of system call trace S2 and S3 (i.e., term $(2,9)$ in term-size 2 and terms $(2,9,9),(1,3,9)$ in term-size 3 representation). Also, we have included a term having all unknown system calls, i.e., $(9,9)$ for termsize 2 and $(9,9,9)$ for term-size 3 . Thus, terms related to unforeseen system calls (i.e., system call number 7 and 8 ) can be handled during testing phase as shown in figure $3 \mathrm{~b}$. Terms $(3,7),(7,3)$ and $(8,5)$ for 2 -gram representation are mapped to term $(9,9)$, while $(2,8)$ is mapped to $(2,9)$. Similarly for 3-gram representation, terms $(3,7,3),(7,3,4)$ and $(4,2,8)$ will be mapped to $(9,9,9)$, while terms $(1,3$, $7)$ and $(2,8,5)$ contribute frequency to term $(1,3,9)$ and term $(2,9,9)$, respectively.

\subsection{Multi-threaded analyzer}

The Analyzer is a multi-threaded instrumentation and decision-making component of our framework. It receives the system call traces from each node (host machine) through a socket. The format of the message received by Analyzer is shown in figure 4. Here, the Process ID is a combination of $\mathrm{CR} 3$ register value and Page Directory Entries (PDE), which uniquely identifies the process. First, Analyzer extracts the IP address information (of cloud 


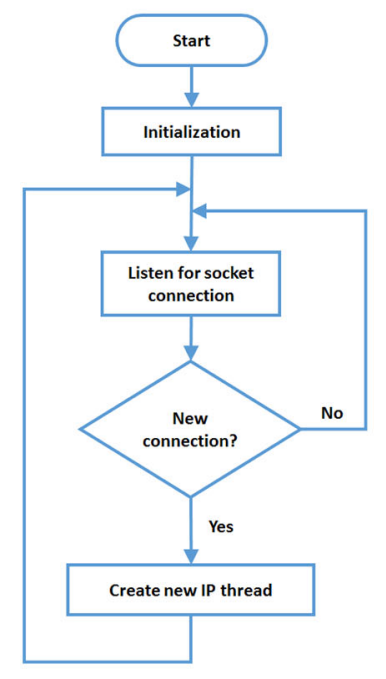

(a)

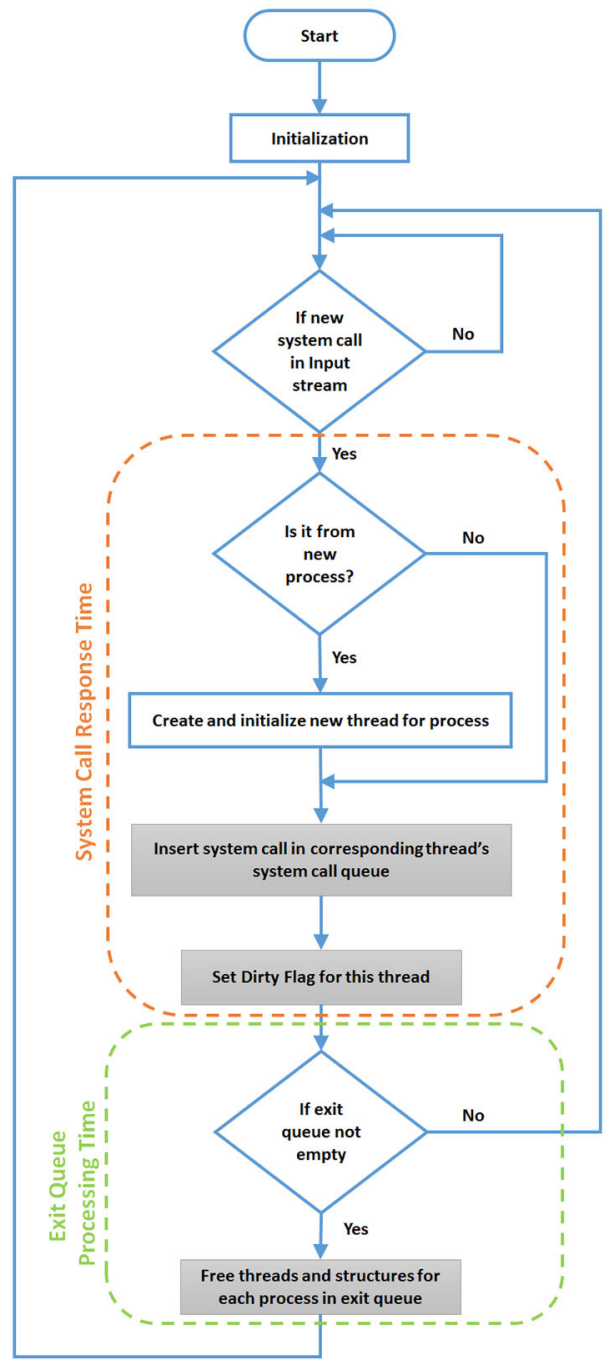

(b)

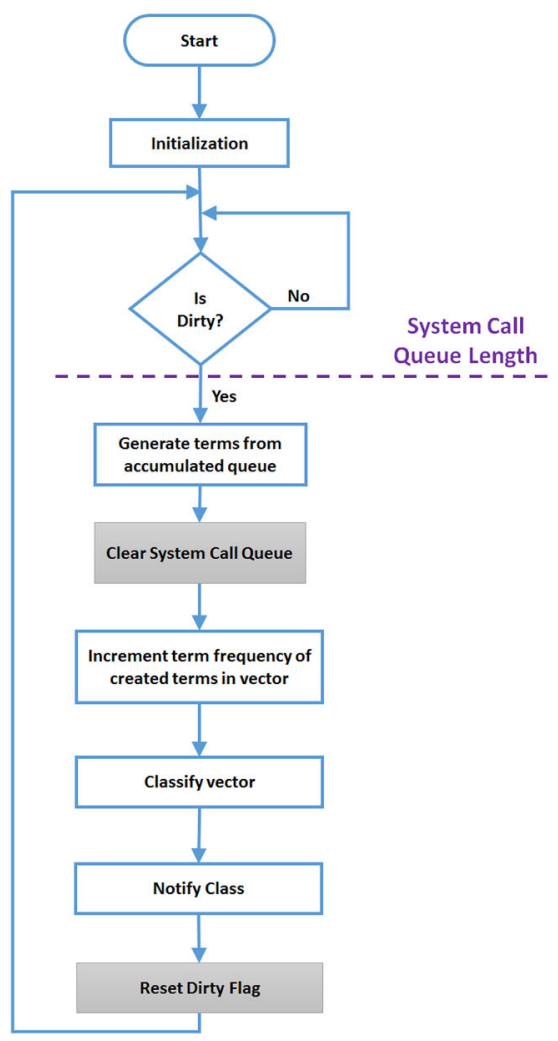

(c)

Figure 6. Work flow of the Analyzer: (a) main() routine, (b) IP-thread and (c) process-thread.

node) from the received message and creates a new thread for that IP (we refer to it as IP-thread), if it is not created before. Each of such IP-threads handles system call traces received from individual cloud nodes. These IP-threads then create and maintain per-process threads (referred to as process-thread) according to a unique pair of VM number and Process ID.

Figure 5 shows the internal working of the IP-thread and process-thread. As shown in the figure, Analyzer follows the following procedures for each IP-thread and each process-thread.

\section{IP-thread}

A1 Receive a message containing IP address, VM number, Process ID and system call value from Nitro running in each node via the Input Queue.

A2 From each received pair, search combinations of VM number and Process ID in Thread Map. (a) If it is not found in the map, create a new entry in the map. Create a new thread for a particular process.

A3 Extract corresponding Thread ID from the map.

A4 Insert the system call value in respective thread's System Call Queue.

A5 Set corresponding Dirty Flag.

A6 If the Exit Queue is not empty, for each Thread ID in Exit Queue,

(a) Clear corresponding entries in Thread Map and other associated structures;

(b) Terminate the thread.

\section{Process-thread}

B1 If Dirty Flag is set, accumulate the collected system calls from thread's System Call Queue and reset the Dirty Flag. 
(a) If the system call is for terminating the process, insert the Thread ID in Exit Queue.

B2 Process the system call numbers and convert them into terms. Update the frequency of all generated terms in the vector.

B3 Predict the class using classification model.

B4 Generate the alert if it is classified as malicious.

Figure 6 shows working of Analyzer in terms of a flow chart. The shaded blocks represent thread-safe operations (assures safe manipulation of shared data among threads), which are achieved using appropriate use of mutexes. As shown in figure $6 \mathrm{a}$, the main() routine continuously listens for new socket connection from cloud nodes. As soon as the new connection is established, the routine creates a new IP thread, which then onwards handles the events of connected cloud node. As shown in figure 6b, each IP-thread handling individual cloud node collects the system call sequence and creates a separate process-thread for individual process ID. Each process-thread generates a feature vector from collected system call sequence and classifies it using a classification model (figure 6c).

\section{Experiments and results}

We use two phases, viz. learning phase and live detection phase, for experiments. Figure 7 shows an abstract view of our system with these phases.

During the learning phase, we process the system call dataset and prepare the feature vectors using a modified vector space model. These feature vectors will be provided as training data to learn the classification model. In the detection phase, we collect the system call traces from Nitro running on each node. These system call traces are then fed into Analyzer component after initial processing. Analyzer component parses the received traces and prepares the feature vectors for respective term-size, which are then used to learn the model. Then these feature vectors will be given to the classifier, which will predict the label. If the label is found to be malicious, the system will generate the alert.

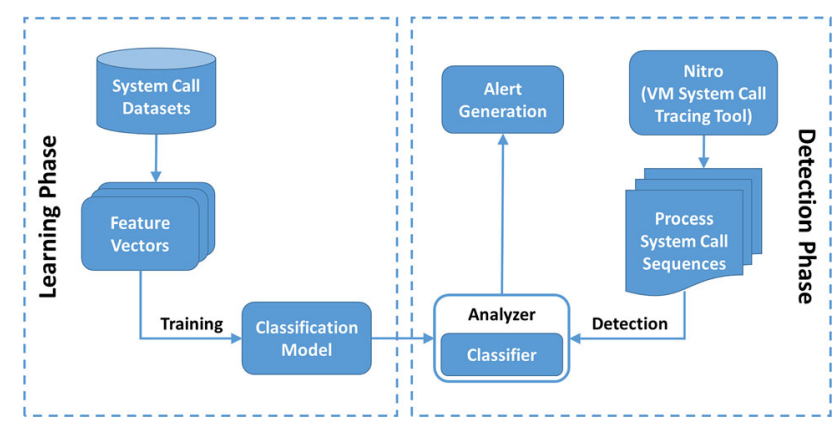

Figure 7. Learning phase and detection phases of VMI-based security framework.

\subsection{Learning phase}

4.1a Datasets and evaluation: To evaluate the applicability of modified vector space representation, we used datasets by Canali et al [33] and Weka workbench $[34,35]$. The datasets (datasets were collected from [36]) contain the execution traces of benign processes and malware of various categories in Windows environment. The execution traces are observed both on synthetic (based on Anubis [37]) and on real machines with actual users and under normal operating conditions. The number of traces in each dataset used for experiments is shown in table 1 along with total number of system calls. These datasets contain process execution traces in the form of 1-gram.

We utilized Weka workbench as a black box tool for evaluation. Weka hosts a number of machine learning algorithms, which can be easily applied on our prepared datasets of varying term-size. We selected nine most common classification algorithms from six different categories given in Weka. The list of selected algorithms, selected options and individual algorithms with their respective category in Weka are described in table 2.

We extract features from the collected datasets and prepare feature vectors in the form of modified vector space

Table 1. Number of traces and system calls in selected datasets.

\begin{tabular}{lcc}
\hline Dataset & Number of traces & Number of system calls \\
\hline Malware & 5,855 & $3,28,99,160$ \\
Goodware & 612 & $65,55,20,685$ \\
Malware-test & 1,133 & $13,18,8452$ \\
Anubis-good & 36 & 44,127 \\
Total & 7,636 & $70,16,52,424$ \\
\hline
\end{tabular}

Table 2. List of selected algorithms with their options.

\begin{tabular}{lcc}
\hline Category & Algorithm & Option selected \\
\hline Bayes & Naïve Bayes & - \\
\hline Function & $\begin{array}{c}\text { Sequential Minimal } \\
\text { Optimization } \\
\end{array}$ & Polynomial kernel \\
& Support Vector & \\
& $\begin{array}{c}\text { Machine } \\
\text { (LibSVM) }\end{array}$ & $\begin{array}{c}\text { Radial Basis Function } \\
\text { (RBF) Kernel, }\end{array}$ \\
& $\begin{array}{c}k \text {-nearest } \\
\text { neighbours (IBk) }\end{array}$ & $k=0.5$, loss $=0.001$ \\
\hline Lazy & Classification via & SimpleKMeans, $k=2$ \\
clustering & Zeta & - \\
\hline Rules & ZeroR & minBucketSize $(\mathrm{B})=5,6,10$ \\
\cline { 2 - 3 } & OneR & Folds $=3$ \\
\cline { 2 - 3 } & JRip & ConfidenceFactor $=0.25$, \\
Trees & J48 &
\end{tabular}




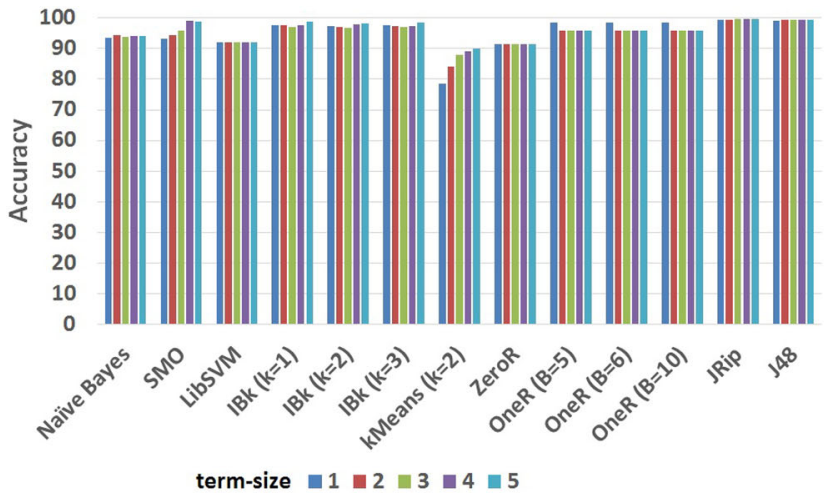

(a)

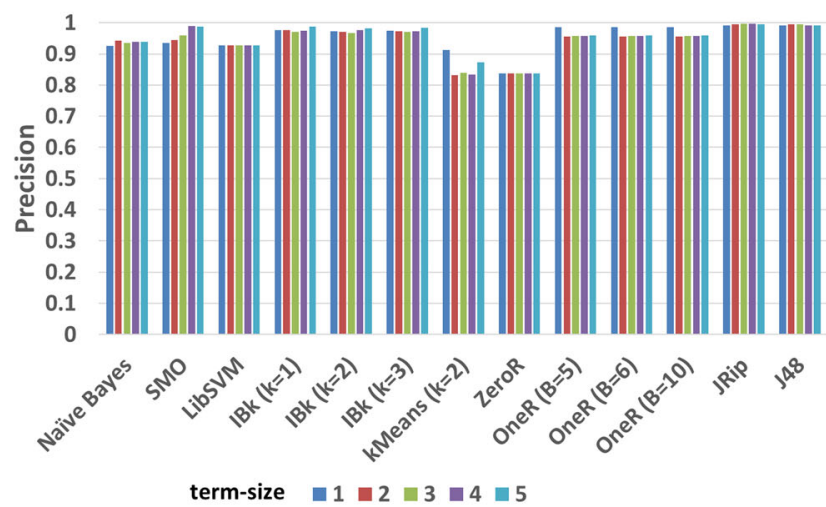

(c)

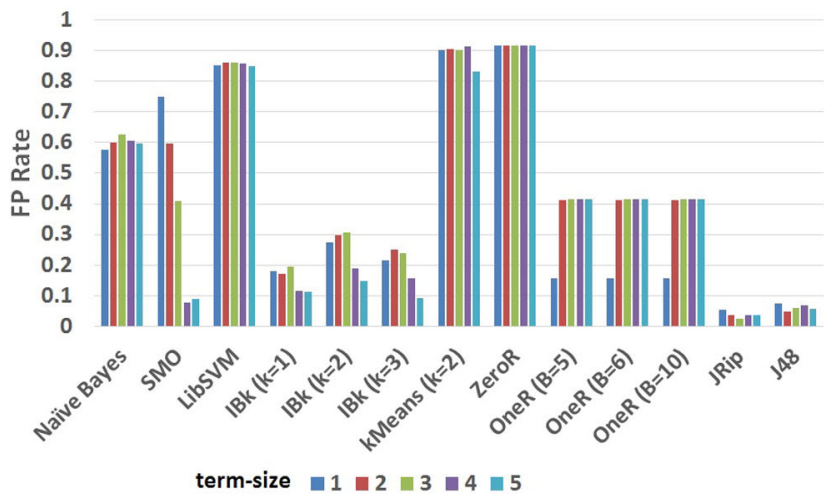

(b)

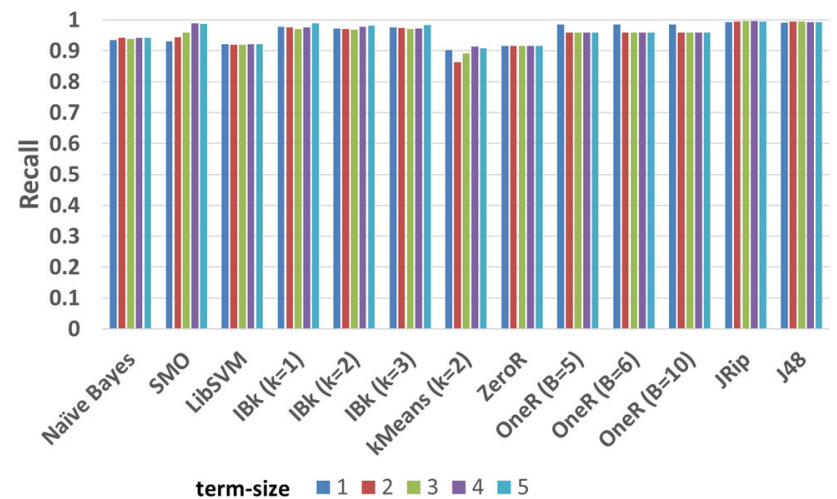

(d)

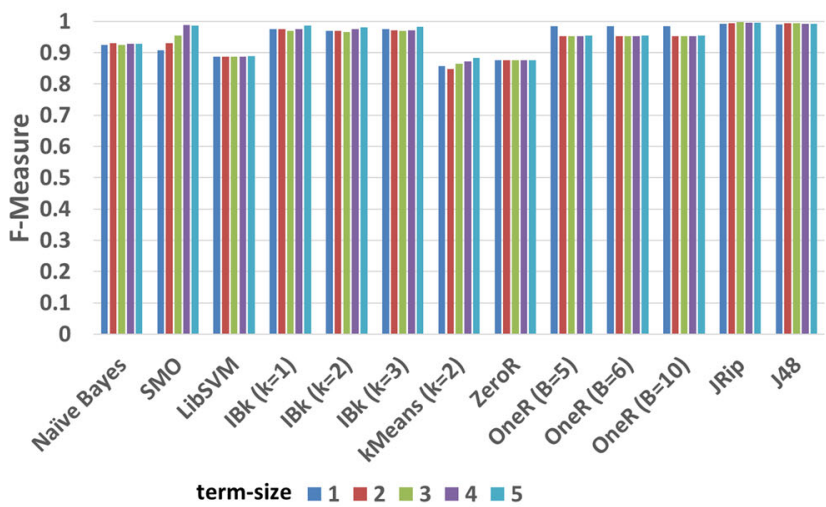

(e)

Figure 8. Performance results of selected algorithms on Anubis system call datasets: (a) accuracy, (b) FP rate, (c) precision, (d) recall and (e) $F$-measure [8].

representation. Then we apply different selected classification algorithms on prepared feature vectors to evaluate performance. We ran each chosen algorithm with selected options on dataset in Weka through a 10-fold cross-validation method.

Figure 8(a)-(e) depicts the accuracy, false positive (FP) rate, precision, recall and $F$-measure results (also reported in [8]) of selected algorithms on datasets, respectively. From the experiment results, we conclude that tree-based algorithm J48 (also known as C4.5 [38]) performs consistently with $99.4 \%$ accuracy (figure $8 \mathrm{a}$ ) and $6.5 \%$ FP rate (figure $8 \mathrm{~b}$ ) for selected datasets. Figure 9 shows the ROC curves of the classifier algorithms used in experiments for varying term-size. From figures 8 and 9 we can depict that J48 and JRip are the closest competitors. Since, the average number of comparisons made for a classification decision is just 5.4 for term-size 3 , we proceed with $\mathrm{J} 48$ as a classification method for 


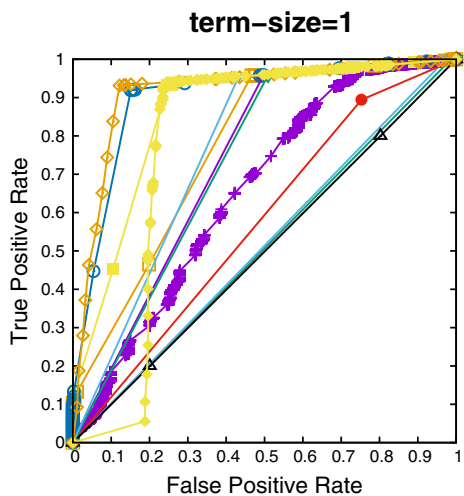

(a)

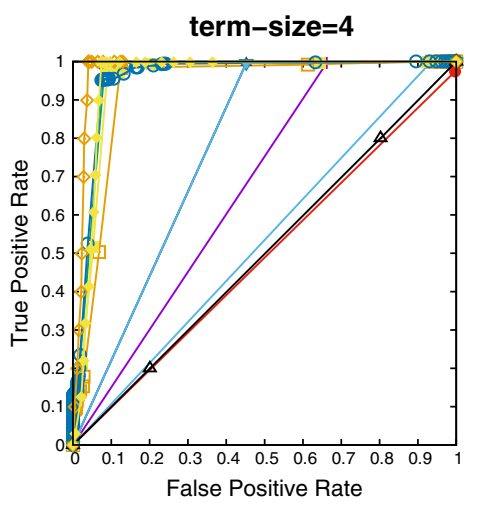

(d)

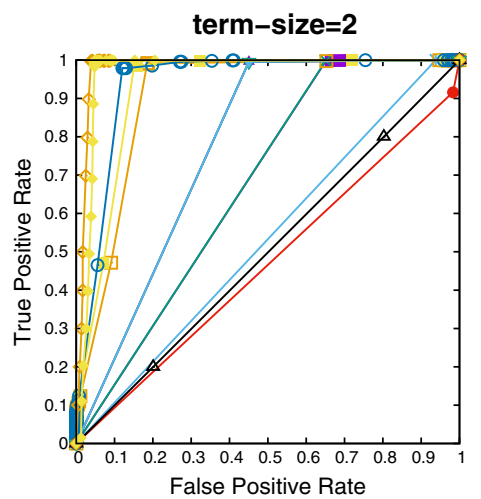

(b)

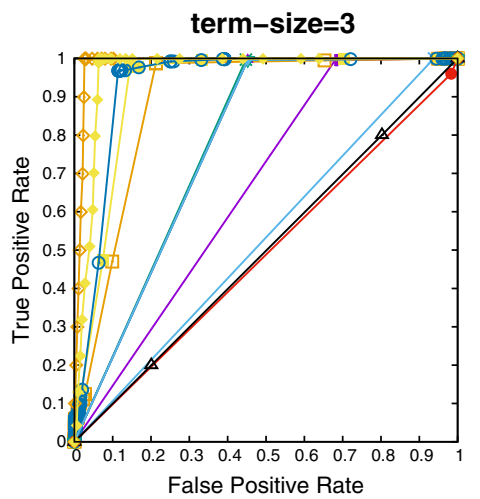

(c)

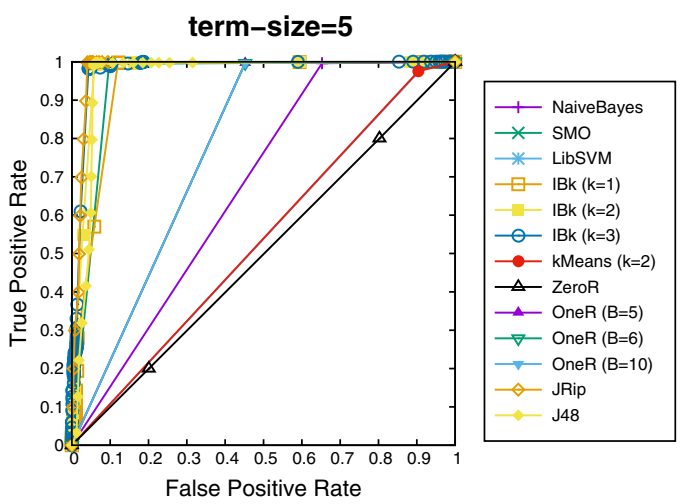

(e)

Figure 9. ROC curves of selected algorithms for varying term-size: $(\mathbf{a})$ term-size $=1$, (b) term-size $=2,(\mathbf{c})$ term-size $=3$, $(\mathbf{d})$ term-size $=4$ and $(\mathbf{e})$ term-size $=5$.

\begin{tabular}{|c|c|c|c|c|c|}
\hline Term & $25,25,119$ & $25,125,167$ & $73,25,125$ & $125,25,173$ & \\
\hline Frequency & 10 & 20 & 4 & 0 & $\ldots \ldots \ldots . . . . . .$. \\
\hline
\end{tabular}

(a)

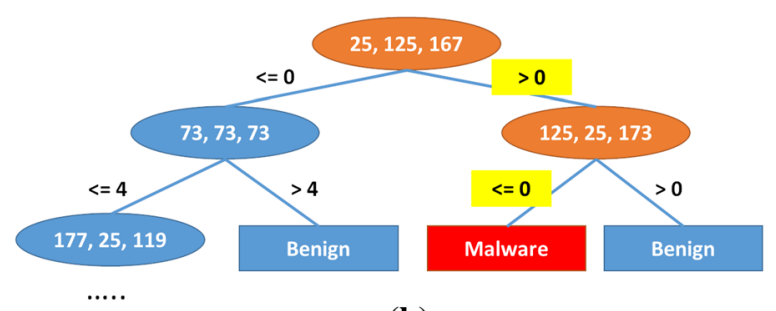

(b)

Figure 10. Process feature vector classification through J48: (a) sample process' feature vector and (b) sample J48 tree model for term-size 3 .

further implementation. It is to be noted that J48 is an open source Java implementation of C4.5 decision tree algorithm in Weka. It chooses the attribute of data that most effectively splits its set of samples into subsets. The splitting criterion is the normalized information gain. The attribute with highest information gain will be chosen to make the decision. This process will be then repeated on smaller subsets. The output of the algorithm is a classification tree. J48-algorithm-based classification model is simple, easy to implement and can be extended according to training data.

Figure 10 describes how feature vectors are classified through a J48 tree. As shown in figure 10a, a given feature vector contains the frequency of individual terms of length (term-size) 3. Figure 10b shows that the J48 decision tree comprises nodes and edges, where nodes represent the terms comprising three system call sequences, while branches represent the decision weight of a given term (its occurrence in a given system call trace) for classification. The leaf node in the tree represents the class label. For classification, the current node term is searched through the feature vector and the corresponding term frequency is determined. The term frequency is compared to edge weight to make a decision on traversing one of the two outgoing edges. This process is repeated until leaf node is reached and the classification label for given feature vector is determined. As shown in figure 10, the sample feature vector path (25, $125,167) \rightarrow(125,25,173) \rightarrow$ malware is traversed according to the frequency of terms given in figure 10a. Hence, the sample process feature vector is classified as a malware. 


\subsection{Live detection phase}

In order to evaluate our framework, we have generated workloads through a benchmark tool PCMark05 [39] used in [25]. It generates various events through a number of tests as listed in table 3 [39].

4.2a Evaluation metrics: Our proposed approach utilizes derivation-based VMI technique for VM monitoring in cloud environment. The proposed multi-threaded Analyzer design is unique on its own. Thus, we use evaluation metrics appropriate for evaluating performance of Analyzer component as follows:

Average response time: This is the time required for an IP-thread to insert a system call into its corresponding

Table 3. List of tests in PCMark05 benchmark.

\section{PCMark05 tests}

\begin{tabular}{lc}
\hline HDD - XP Startup & Video Encoding \\
Physics \& 3D & Audio Compression \\
2D Transparent Windows & Text Edit \\
3D Pixel Shader & Image Decompression \\
Web Page Rendering & File Compression \\
File Decryption & Memory Latency \\
2D Graphics Memory 64 Lines & HDD - Virus \\
& Scanning \\
HDD - General Usage & File Encryption \\
\hline
\end{tabular}

Process-thread's System Call Queue. This primarily measures the performance of the IP-thread.

Average system call queue length: This is the average number of system calls that are waiting in System Call Queue between process classifications. This primarily measures the performance of the Process-thread.

Average exit queue processing time: This is the average time required to process the Exit Queue. The Exit Queue contains thread IDs, whose related processes in VMs are terminated. This involves removing each thread's entry in global data structures and deleting the thread itself.

4.2b Experimental set-up: We have considered Eucalyptus cloud architecture for implementation. Eucalyptus [40, 41] is a free and open-source framework for building private and hybrid cloud. It implements IaaS and facilitates users an ability to run and control VM instances, deployed across various physical resources.

Nitro extends the KVM to support VM system call trapping. Thus, we replace QEMU and KVM modules of each node controller in Eucalyptus cloud setup with user space and kernel space modules of Nitro. This enables trapping of system calls of VMs hosted on the node controller.

Our experiments are conducted using set of five host machines (as node controllers) and a separate Analyzer host machine. The set of host machines include three units of HP ProLiant DL120G6 rack server and two units of Dell

Table 4. Performance results of experiments (with standard deviation of $2.42 \%, 3.16 \%$ and $4.30 \%$ for Average Response Time, Average Queue Length and Average Exit Queue Processing Time respectively).

\begin{tabular}{|c|c|c|c|c|c|}
\hline \multirow[b]{2}{*}{ No. of hosts } & \multicolumn{5}{|c|}{ No. of VMs per host } \\
\hline & 1 & 2 & 3 & 4 & 5 \\
\hline \multicolumn{6}{|c|}{ Average response time ( $\mu \mathrm{s})$} \\
\hline 1 & 171.4583 & 273.5050 & 308.7123 & 497.5357 & 875.8867 \\
\hline 2 & 393.3180 & 535.8300 & 1097.5730 & 1968.9500 & 3233.9070 \\
\hline 3 & 670.5810 & 1506.9070 & 2123.3130 & 3142.3230 & 5293.4270 \\
\hline 4 & 1146.6570 & 1966.5600 & 3353.8900 & 5329.3270 & 11104.0300 \\
\hline 5 & 2161.7230 & 5062.2730 & 8471.0830 & 12778.0300 & 16485.2000 \\
\hline \multicolumn{6}{|c|}{ Average queue length } \\
\hline 1 & 84.5989 & 113.6147 & 118.5947 & 106.9597 & 89.7392 \\
\hline 2 & 179.8713 & 157.0267 & 125.8340 & 108.9777 & 92.8244 \\
\hline 3 & 211.7717 & 158.7543 & 127.7970 & 114.9903 & 93.1897 \\
\hline 4 & 225.1947 & 159.1860 & 131.5773 & 100.6157 & 95.0283 \\
\hline 5 & 234.2400 & 161.8530 & 140.5360 & 125.0932 & 102.2487 \\
\hline \multicolumn{6}{|c|}{ Average exit queue processing time $(\mu \mathrm{s})$} \\
\hline 1 & 11.2167 & 11.7476 & 14.8883 & 16.7484 & 24.7817 \\
\hline 2 & 13.9158 & 15.1905 & 20.8002 & 25.5029 & 32.6808 \\
\hline 3 & 17.9041 & 19.7875 & 26.4005 & 34.10403 & 38.0561 \\
\hline 4 & 18.7445 & 24.9128 & 33.1067 & 37.8569 & 49.1430 \\
\hline 5 & 29.01147 & 33.77533 & 49.7412 & 57.9837 & 65.9051 \\
\hline
\end{tabular}


OPTIPLEX 990. The configuration of HP ProLiant DL120G6 is an Intel Xeon 4 Core processor, 8-GB RAM and 1-TB hard disk. Dell OPTIPLEX 990 has an Intel Core i5 processor, 4-GB RAM and 500-GB hard disk. Each machine runs a 64-bit Ubuntu OS. We created a Windows XP guest VM having PCMark05 benchmark installed in it. The Analyzer is placed inside a VM on separate host machine with size of 8 CPU core and 10-GB RAM. This separate host machine is an IBM Blade Server with 96-GB RAM and 16-core processor.

We evaluate the performance of Analyzer to check scalability by gradually increasing number of running VMs on a host. We increase number of physical hosts to check the performance of Analyzer under varying loads. For each evaluation metric, we run a benchmark in VM, extract the system call traces through Nitro and send them to Analyzer for classification. We repeat this process for a number of VMs and hosts, running the benchmark in parallel inside each VM.

4.2c Results and discussion: Table 4 summarizes the results of experiments (executing the benchmark) with number of hosts running number of VMs. Trends for the same are presented in figure 11. For the purpose of following discussions, we shall refer to IP-thread as a writer thread (it "writes" into system call queue) and process-thread as a reader thread (it "reads" and empties system call queue). We discuss observed results as follows with respect to defined evaluation metrics.

Average response time: As the number of hosts and/or VMs increases, it induces a delay in writing a processspecific system call by the writer thread. This in turn increases the average response time, as evident from figure 11a.

Average system call queue length: As number of VMs increases, the overall number of threads being scheduled by the Analyzer increases. Thus, the wait time for the writer thread increases, which reduces the rate of system calls being "written" to a per-process system call queue. Thus the average queue length decreases - VM axis in figure $11 \mathrm{~b}$.

As the number of hosts increases, the number of writer threads increases, thereby increasing the average system call queue length - host axis in figure $11 \mathrm{~b}$.

The anomalous trend corresponding to number of hosts equal to 1 can be explained by considering the number of CPU cores available with the Analyzer. Given our setting, the number of threads (corresponding to the situation with low number of VMs) was scheduled extremely fast on the multicore CPU running the Analyzer.

Average exit queue processing time: Exit queue holds the thread IDs of corresponding terminated processes, which were running inside VMs. It is processed by IP-thread. As we ran the benchmark in multiple VMs hosted on multiple hosts, many threads were created and terminated during the

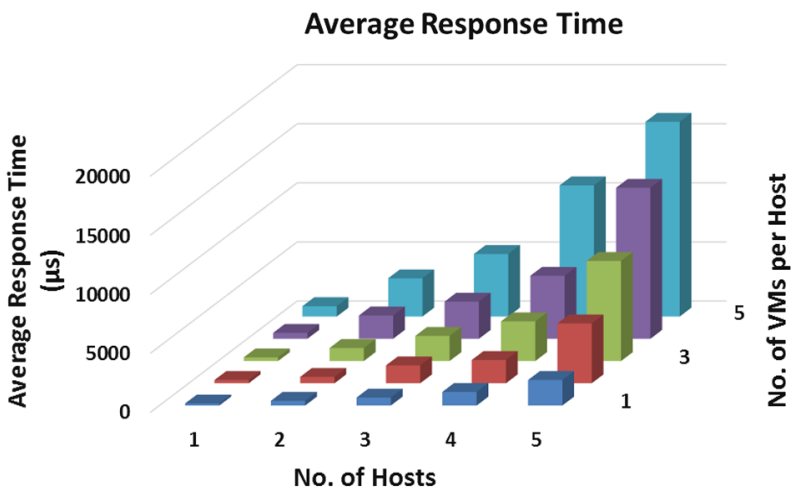

(a)

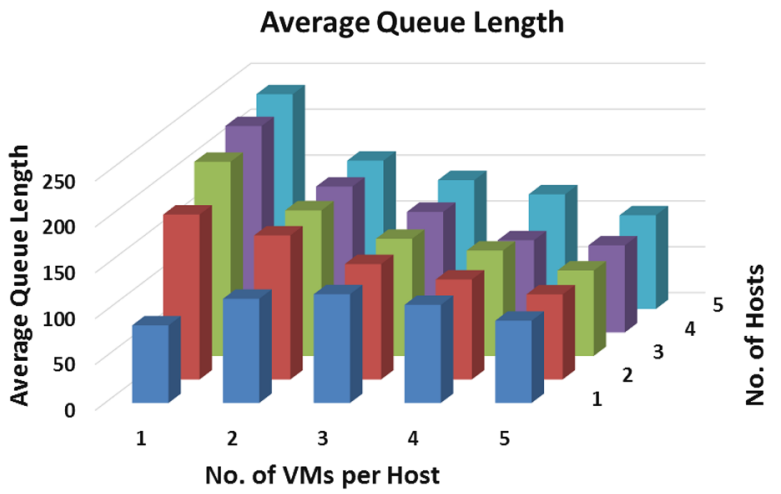

(b)

\section{Average Exit Queue Processing Time}

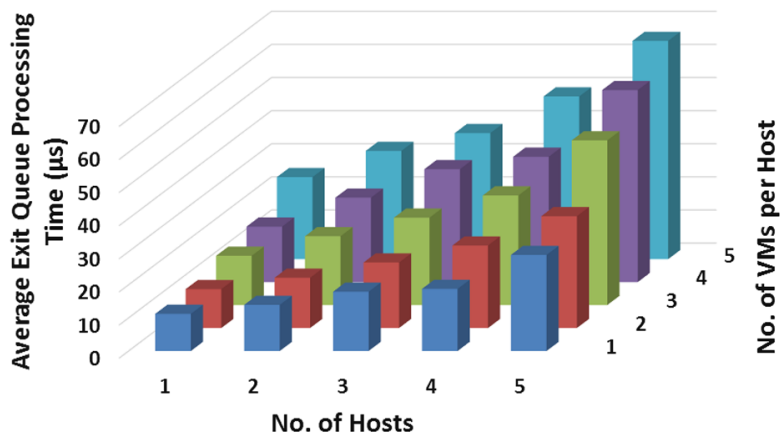

(c)

Figure 11. Results of evaluation: (a) average response time, (b) average queue length and (c) average exit queue processing time.

process. Exit queue processing time increased considerably with increase in number of VMs and hosts, as seen in figure $11 \mathrm{c}$.

The minimum average response time for one host running one $\mathrm{VM}$ is $0.17 \mathrm{~ms}$, while the maximum average response time during relatively heavy load (five physical machines each running five VMs) is $16.5 \mathrm{~ms}$. For a cloud network, hosting hundreds of VMs, response time will be too high. To process requests from large number of VMs in real time, one can place multiple Analyzers that can work 
Table 5. Performance results of experiments with two Analyzers.

\begin{tabular}{|c|c|c|c|c|}
\hline \multicolumn{2}{|r|}{ Analyzer 1} & \multicolumn{2}{|r|}{ Analyzer 2} & \multirow[b]{2}{*}{ Maximum average response time $(\mu \mathrm{s})$} \\
\hline No. of hosts & Average response time $(\mu s)$ & No. of hosts & Average response time $(\mu s)$ & \\
\hline 5 & 16485.2000 & 0 & NA & 16485.2000 \\
\hline 4 & 11127.3500 & 1 & 884.8865 & 11127.3500 \\
\hline 3 & 5374.1700 & 2 & 3209.4900 & 5374.1700 \\
\hline
\end{tabular}

Table 6. Malware detection results in Live Detection Phase.

\begin{tabular}{lccc}
\hline Sample & $\begin{array}{c}\text { Total } \\
\text { samples }\end{array}$ & $\begin{array}{c}\text { Detected as } \\
\text { benign }\end{array}$ & $\begin{array}{c}\text { Detected as } \\
\text { malware }\end{array}$ \\
\hline Benign & 22 & 21 & 1 \\
Worm & 47 & 1 & 46 \\
Trojan & 88 & 6 & 82 \\
\hline
\end{tabular}

individually to handle load from allotted cloud servers. We also performed experiments with two Analyzers. For these experiments, system configuration of second Analyzer is the same as the first one. We performed the experiments by distributing the load of number of hosts (total five hosts) running five VMs between both Analyzers. Table 5 shows the experiment results with two Analyzer. As shown in the table, we provide the average response time for individual Analyzers. As both Analyzers run in parallel, maximum average response time will be the measure for combined average response time. As evident from the results, maximum response time decreases as we distribute the input load among Analyzers.

We tested the live detection phase working with malware samples. We collected malware samples found in the wild, which comprise worms and trojans. We train the classifier with Anubis dataset (comprising malware and benign process traces) and system call traces collected from VM in idle condition and running PCMark05 benchmark tool (labelling as benign processes). We ran VM for $24 \mathrm{~h}$ and collected 25,98,214 system calls for idle session using Nitro. By running PCMark05 we collected 21,02,915 system calls. Table 6 summarizes the experiment results for malwares executed inside VM. The result shows that the malwares are detected with $94.90 \%$ accuracy, $94.81 \%$ true positive rate and $4.54 \%$ FP rate.

\section{Conclusion}

We presented VMI-based security framework for cloud. It uses derivation-based view generation pattern for VMI. It is capable of detecting in-VM malicious activities from outside VM without providing any external semantic knowledge. We derived the process classification features from extracted in-VM process' system call traces and described them in our modified vector space model [8]. The proposed framework was designed specifically for scenario of multiple VMs running multiple processes hosted on multiple physical machines. We presented the appropriate evaluation metrics to evaluate our multi-threaded architecture design. The experimental results demonstrate that the proposed multi-threaded architecture design can handle system call traces of a number of VMs, hosted on multiple nodes efficiently. To achieve real time performance, one can utilize multiple Analyzers. Thus, the proposed framework is suitable for classifying in-VM activities in cloud environment.

\section{References}

[1] Clark K, Warnier M and Brazier F 2011 BOTCLOUDS - the future of cloud-based Botnets? In: Proceedings of the 1st International Conference on Cloud Computing and Services Science, SciTePress, pp. 597-603

[2] Comazzetto A 2011 Botnets: the dark side of cloud computing. SOPHOS Technical Report, Bostan, USA, https:// cloud.report/Resources/Whitepapers/4762db4d-c561-4f62bd3f-9eee93843cb7_Sophos.pdf

[3] Modi C, Patel D, Borisaniya B, Patel H, Patel A and Rajarajan M 2013 A survey of intrusion detection techniques in Cloud. J. Netw. Comput. Appl. 36(1): 42-57

[4] Pfoh J, Schneider C and Eckert C 2009 A formal model for virtual machine introspection. In: Proceedings of the 1st ACM Workshop on Virtual Machine Security, ACM VMSec'09, pp. 1-10

[5] Bahram S, Jiang X, Wang Z, Grace M, Li J, Srinivasan D, Rhee J and Xu D 2010 DKSM: Subverting virtual machine introspection for fun and profit. In: Proceedings of the 29th IEEE Symposium on Reliable Distributed Systems, pp. 82-91

[6] Pfoh J, Schneider C and Eckert C 2011 Nitro: hardwarebased system call tracing for virtual machines. In: Iwata $\mathrm{T}$ and Nishigaki M (Eds.) Advances in Information and Computer Security, Lecture Notes in Computer Science, vol. 7038. Springer, Berlin, Heidelberg, pp. 96-112

[7] Borisaniya B and Patel D 2014 Evasion resistant intrusion detection framework at Hypervisor layer in Cloud. In: Proceedings of the International Conference on Advances in Communication, Network, and Computing, CNC 2014, pp. 748-756

[8] Borisaniya B, Patel K and Patel D 2014 Evaluation of applicability of modified vector space representation for inVM malicious activity detection in Cloud. In: Proceedings of the Annual IEEE India Conference (INDICON), pp. 1-6 
[9] Dolan-Gavitt B, Leek T, Zhivich M, Giffin J and Lee W 2011 Virtuoso: narrowing the semantic gap in virtual machine introspection. In: Proceedings of the 2011 IEEE Symposium on Security and Privacy (SP), pp. 297-312

[10] Payne B D, de Carbone M and Lee W 2007 Secure and flexible monitoring of virtual machines. In: Proceedings of the Twenty-Third Annual Computer Security Applications Conference, ACSAC 2007, pp. 385-397

[11] Payne B D 2012 Simplifying virtual machine introspection using LibVMI. Sandia Report, September 2012, http:// prod.sandia.gov/techlib/access-control.cgi/2012/127818. pdf

[12] Schneider C, Pfoh J and Eckert C 2012 Bridging the semantic gap through static code analysis. In: Proceedings of the 5th European Workshop on System Security (EuroSec 2012)

[13] Fu Y and Lin Z 2012 Space traveling across VM: automatically bridging the semantic gap in virtual machine introspection via online kernel data redirection. In: Proceedings of the IEEE Symposium on Security and Privacy (SP), pp. 586-600

[14] Newsome J and Song D 2005 Dynamic taint analysis for automatic detection, analysis, and signature generation of exploits on commodity software. In: Proceedings of the Network and Distributed Systems Security Symposium (NDSS'05)

[15] Saberi A, Fu Y and Lin Z 2014 Hybrid-BRIDGE: efficiently bridging the semantic gap in virtual machine introspection via decoupled execution and training memoization. In: Proceedings of the Network and Distributed Systems Security Symposium (NDSS14)

[16] Michie D 1968 Memo functions and machine learning. $\mathrm{Na}$ ture 218(5136): 19-22

[17] Dinaburg A, Royal P, Sharif M and Lee W 2008 Ether: malware analysis via hardware virtualization extensions. In: Proceedings of the 15th ACM Conference on Computer and Communications Security (CCS '08), pp. 51-62

[18] Dinaburg A, Royal P, Sharif M and Lee W Ether. http:// ether.gtisc.gatech.edu/

[19] Ibrahim A S, Hamlyn-Harris J, Grundy J and Almorsy M 2011 CloudSec: a security monitoring appliance for virtual machines in the IaaS cloud model. In: Proceedings of the 5th International Conference on Network and System Security (NSS), pp. 113-120

[20] VMware VMsafe. http://www.vmware.com/company/news/ releases/vmsafe_vmworld.html

[21] Baek H W, Srivastava A and Van der Merwe J 2014 CloudVMI: virtual machine introspection as a Cloud service. In: Proceedings of the IEEE International Conference on Cloud Engineering (IC2E), pp. 153-158

[22] Yao F, Sprabery R and Campbell R H 2014 CryptVMI: a flexible and encrypted Virtual Machine Introspection system in the Cloud. In: Proceedings of the 2nd International Workshop on Security in Cloud Computing (SCC), pp. 11-18

[23] Suneja S, Isci C, Bala V, de Lara E and Mummert T 2014 Non-intrusive, out-of-band and out-of-the-box systems monitoring in the Cloud. In: Proceedings of the 2014 ACM International Conference on Measurement and Modeling of Computer Systems (SIGMETRICS), pp. 249-261
[24] Forrest S, Hofmeyr S, Somayaji A and Longstaff T 1996 A sense of self for Unix processes. In: Proceedings of the IEEE Symposium on Security and Privacy, pp. 120-128

[25] Pfoh J, Schneider C and Eckert C 2013 Leveraging string kernels for Malware detection. In: Proceedings of the 7th International Conference on Network and System Security, Lecture Notes in Computer Science, vol. 7873. Springer, Berlin, Heidelberg, pp. 206-219

[26] Lodhi H, Saunders C, Shawe-Taylor J, Cristianini N and Watkins C 2002 Text classification using string kernels. J. Mach. Learn. Res. 2: 419-444

[27] Wang X, Yu W, Champion A, Fu X and Xuan D 2007 Detecting worms via mining dynamic program execution. In: Proceedings of the Third International Conference on Security and Privacy in Communications Networks and the Workshops (SecureComm), pp. 412-421

[28] Kang D K, Fuller D and Honavar V 2005 Learning classifiers for misuse and anomaly detection using a bag of system calls representation. In: Proceedings from the Sixth Annual IEEE SMC Information Assurance Workshop, (IAW 2005), pp. 118-125

[29] Rieck K, Holz T, Willems C, Düssel P and Laskov P 2008 Learning and classification of Malware behavior. In: Proceedings of the 5th International Conference on Detection of Intrusions and Malware, and Vulnerability Assessment, Lecture Notes in Computer Science, vol. 5137. Springer, Berlin, Heidelberg, pp. 108-125

[30] Liao Y and Vemuri V R 2002 Using text categorization techniques for intrusion detection. In: Proceedings of the 11th USENIX Security Symposium, Berkeley, CA, USA, vol. 12, pp. 51-59

[31] Wagner D and Dean D 2001 Intrusion detection via static analysis. In: Proceedings of the 2001 IEEE Symposium on Security and Privacy, pp. 156-168

[32] Wagner D and Soto P 2002 Mimicry attacks on host-based intrusion detection systems. In: Proceedings of the 9th ACM Conference on Computer and Communications Security (CCS), pp. 255-264

[33] Canali D, Lanzi A, Balzarotti D, Kruegel C, Christodorescu M and Kirda E 2012 A quantitative study of accuracy in system call-based malware detection. In: Proceedings of the 2012 International Symposium on Software Testing and Analysis (ISSTA), pp. 122-132

[34] Weka. http://www.cs.waikato.ac.nz/ml/weka/

[35] Holmes G, Donkin A and Witten I H 1994 Weka: a machine learning workbench. In: Proceedings of the 1994 Second Australian and New Zealand Conference on Intelligent Information Systems (ANZIIS), pp. 357-361

[36] Canali D Anubis System call Dataset. http://s3.eurecom.fr/ $\sim$ canali/resources.html

[37] Anubis - malware analysis for unknown binaries. https:// anubis.iseclab.org/

[38] Quinlan J 1993 C4.5: Programs for machine learning. In: Morgan Kaufmann Series in Machine Learning. Morgan Kaufmann Publishers Inc. San Francisco, CA, USA

[39] Sami N 2005 PCMark 05 PC performance analysis - white paper. June 2005, http://s3.amazonaws.com/download-aws. futuremark.com/pcmark05-whitepaper.pdf

[40] Nurmi D, Wolski R, Grzegorczyk C, Obertelli G, Soman S, Youseff L and Zagorodnov D 2008 Eucalyptus: a technical report on an elastic utility computing archietcture linking 
your programs to useful systems. UCSB Computer Science Technical Report, pp. 1-16

[41] Nurmi D, Wolski R, Grzegorczyk C, Obertelli G, Soman S, Youseff L and Zagorodnov D 2009 The Eucalyptus open- source cloud-computing system. In: Proceedings of the 9th IEEE/ACM International Symposium on Cluster Computing and the Grid (CCGRID 2009), pp. 124-131 